\title{
Reversible Watermarking with FPGA Implementation
}

\author{
V. Swathy ${ }^{1}$, K. Vijayalakshmi ${ }^{2}$, B. Senthil Raja ${ }^{3}$ \\ PG Scholar, Department of ECE (PG), SKP Engineering College, Tiruvannamalai, India ${ }^{1}$ \\ Associate Professor, Department of ECE (PG), SKP Engineering College, Tiruvannamalai, India ${ }^{2}$ \\ Assistant Professor Department of ECE (PG), CK College of Engineering and Technology, Cuddalore, India ${ }^{3}$
}

\begin{abstract}
Watermarking is an important field for copyrights of digital media and owner authentication. Reversible image watermarking method based on contrast mapping accomplishes different transform applied on pixels and their least significant bits are used for data embedding. It is invertible if the least significant bits of the transformed pixels are lost during data embedding. Reversible contrast mapping offers high embedding data at low visual distortion. Distortion caused due to embedding should be made small. This lead to the original and the watermarked image equivalent so that embedded data remains imperceptible on visualization. This type of imperceptible distortion is not acceptable in medical and military image. This has led to an interest on reversible watermarking, where the embedded watermark is not only extracted but also perfect restoration of the original signal is possible from the watermarked image. Low computation cost and ease of semicustom make it attractive for real time implementation. The design and implementation of a FPGA (Field Programmable Gate Array) based architecture using image watermarking algorithm is done on host image. Security level offered by the watermarking techniques based on hardware is higher than the software based watermarking techniques. Distortion caused due to embedding should be made small as much as possible, so distortion control technique is used based on threshold values.
\end{abstract}

Keywords: Reversible image watermarking, Reversible contrast mapping, Semicustom, Secret image.

\section{INTRODUCTION}

Digital watermarking is expressed as a knowledge concealing technique that is developed for functions like identification, copyright protection and classification of digital media content. During this technique, a secret information referred to as watermark is embedded into the digital transmission content with in the decoder, watermark information is extracted from the watermarked signal in an exceedingly loss less manner though original signal can't be obtained back. In some necessary applications like military notional, rhetorical law and medical notional, distortion within the original signal might cause fatal results. For instance, a tiny low distortion in an exceedingly medical image might interfere with the accuracy of document identification. Distortion issues which can arise in an exceedingly applications will be fastened in a reversible watermarking technique. Reversible image watermarking algorithms can be divided into five groups. Lossless compression based algorithms, difference expansion based algorithms, and histogram shifting based algorithms, prediction error expansion based algorithms and integer to integer transform based algorithms. Performance of a watermarking algorithm is categorized into three parts. They are visual quality, payload capacity and computational complexity.

A hardware implementation can be designed on a field programmable gate array board or custom integrated circuit. The difference between FPGA and custom IC implementation is a trade-off among the cost, power consumption and performance. Hardware implementation using FPGA has advantages of low investment cost, simpler design cycle, field programmability and desktop testing with medium processing speed. On the other side, due to lower unit cost, full custom capability and from an integration point, custom implementation application specific integrated circuit design may be more useful. During past years, FPGAs were selected primarily for lower speed, complexity, volume designs, but today's FPGAs can easily push upto the $500 \mathrm{MHz}$ performance barrier.

A literature survey is survived for various papers which are important to know the previously available techniques and their advantages and limitations. It also includes the various supporting papers for the proposed technique and their advantages. There are many techniques available for reversible image watermarking. The reversible contrast mapping method provides to embed and extract the watermarking. The data of secretly hiding and communicating information has gained immense importance in the two decades due to the advantages in generation, storage, and communication technology of digital content. Watermarking is solutions for tamper detection and protection of digital content. Watermarking can cause damage to the information present in the cover work. 


\section{ISO 3297:2007 Certified}

Vol. 5, Issue 5, May 2017

At the receiving end, the exact reconstruction of the work may not be possible. In addition there exist certain applications that may not pass even small distortions in report work priority to the downstream techniques. In those applications, reversible watermarking instead of other watermarking is occupied. Reversible watermarking of digital image allows full extraction of the watermark along with the complete reconstruction of the cover work. In past years, reversible watermarking process is gaining popularity because of its increasing applications in important and sensitive areas, i.e., military information, health care, and law-enforcement. Due to the rapid evolution of reversible watermarking process, Latest survey of recent research in this field is highly desirable. Security level offered by the watermarking techniques based on hardware is higher than the software based watermarking techniques.

A reversible watermarking technique without using modulo addition was then introduced by proposed the concept of compressing the least significant bit plane of cover image to space for the watermark to be embedded. From the Fig 1 the watermark bits are embedded in the unsampled pixels until no unsampled pixel is left. After that, the remaining of the watermark bits are inserted into the sampled pixels, which are interpolated using nearby watermarked pixels. This scheme provides low embedding distortion and less computational cost, which result in good image quality and efficient algorithm. But the pixels having value 0 or 255 are unconsidered for embedding in order to prevent overflow/underflow. Correlation between adjacent pixels is efficiently uncorrelated with interpolation process. Embedding capacity is improved by adaptive embedding algorithm. Pixel selection process is utilized to obtain good visual quality. Interpolation value is estimated by using enclosing pixels to obtain better prediction. Structural similarity index is another metric which measures the similarity between different images. The original image is partitioned into $8 \times 8$ or $32 \times 32$ non-overlapping block of pixels. Later on each block is partitioned into pairs of pixel values.

a. Read cover image

b. Read watermark image

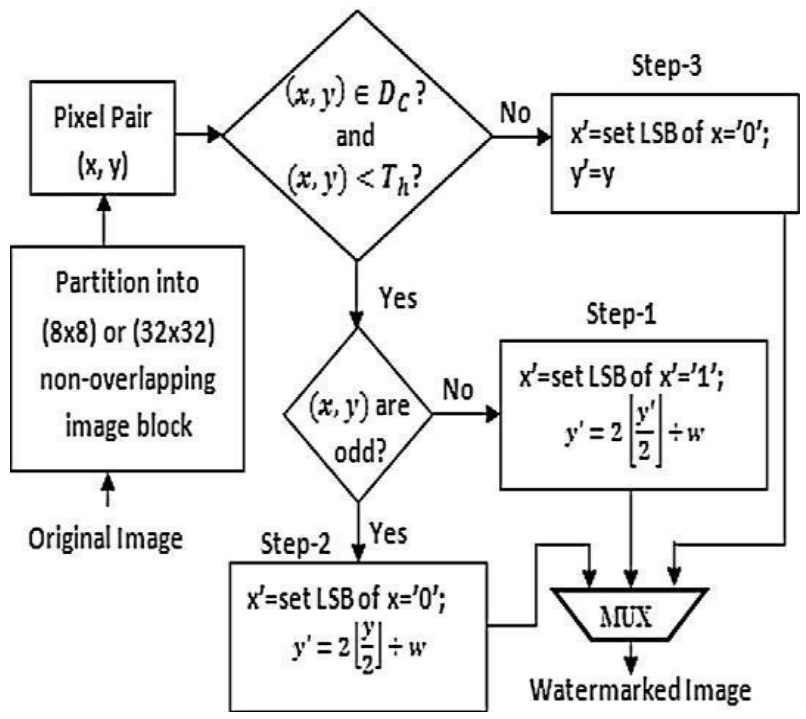

Fig 1. Dataflow diagram of watermark embedding

\section{REVERSIBLE CONTRAST MAPPING ALGORITHM}

Let $(x, y)$ be the values of pixel pair in an image, then the pixel intensity values are bounded between [0, 255] for an 8 bits or pixel gray scale image. The forward integer transform for a pair of pixel values is defined. To prevent the overload and the under load problem, the transform pair is restricted within a sub-domain. The inverse transformation can perfectly restored the pixel pair values even if the least significant bits of the transformed pixel pairs are lost, except when both pair of pixels is odd set of values.

The occurrence of odd pair of pixels is expected to below with respect to the total possibility of occurrence of other combinations; hence a large set of pair of pixels may be available for data embedding. So that reversible contrast mapping provides high embedding bit rate and this is achieved at a very low mathematical complexity.

High payload embedding through passing more data insertion introduced many visual distortions. Distortion control is needed to reduce recognized degradation. A straight forward attempt to control such distortion is to transform pair of pixel values only if they do not exceed a predefined error threshold or distortion threshold. 


\section{WATERMARK EMBEDDING}

To realize reversible contrast mapping algorithm, the original image is partitioned into non overlapping groups of pixels pairs following either horizontally or vertically, like any space filling curve. Aim of this work is to develop semicustom,

Partition watermark image into $32 \times 32$ blocks

Partition watermark image into $32 \times 16$ blocks

Mapping

Divide pixel pairs into different sets

Initialize various index values

Set S1 contains all pixels that satisfy the difference value condition

Set S2 contains all expandable pixel pairs tha are not in S1

Set S21 contains all pixel pairs whose difference value is less than threshold

$\checkmark$ Set S22 contains all pixel pairs whose difference value is greater than threshold

Set S3 contains all changeable pixel pairs embedding is performed in st S3

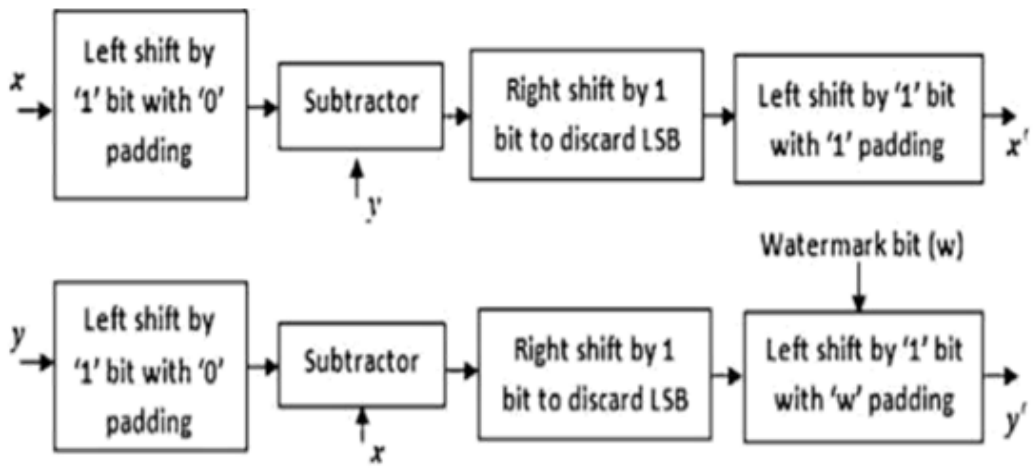

Fig 2. Data path for step-1 of watermark embedding

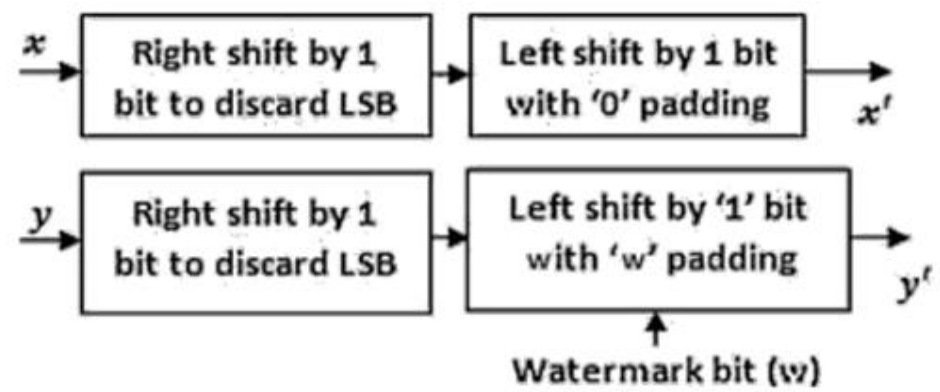

Fig 3. Data path for step-2 of watermark embedding

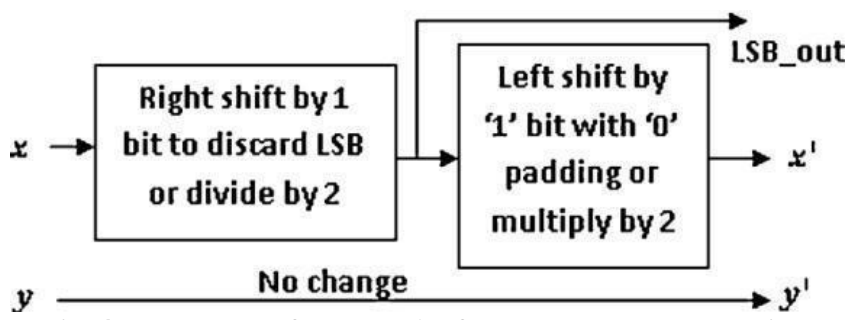

Fig 4. Data path for step-3 of watermark embedding

Fig 2 indicates that the resultant value is left shifted by 1 bit with 1 padding. Similarly the watermark bit is embedded into the LSB of y. Fig 3 indicates the Step- 2 operation of watermark embedding, where LSB of $\mathrm{x}$ is made 0 by two consecutive shifting operations. The value of $\mathrm{x}$ is first right shifted by 1 bit to discard its LSB, then 1 bit left shifting operation with 0 padding is performed to generate the final result. In the similar way watermark data is embedded into the LSB of y. Fig 4 indicates that Step-3, is the simplest among the three. 


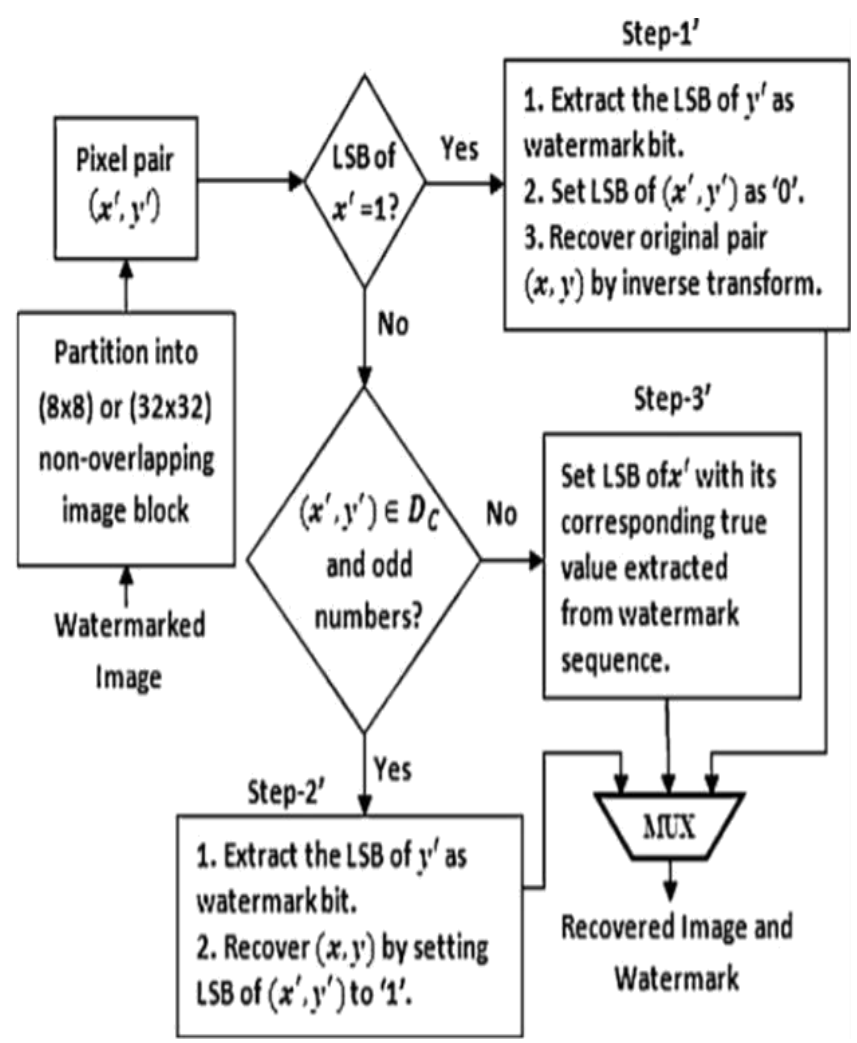

Fig 5. Data flow diagram of watermark extraction

\section{WATERMARK EXTRACTION}

Watermark extraction algorithm:

1. Divide the received image into several $2 \times 1$ segments $(X, Y)$

2. Classify if $X^{\prime}$ is an even or odd number and extract the message with the following three strategies:

a) If $\mathrm{X}^{\prime}$ is an odd number, extract the LSB of $\mathrm{Y}^{\prime}$ and that is the embedded message. In order to recover the image, we replace the $\mathrm{LSB}$ of $\mathrm{X}^{\prime}$ and $\mathrm{Y}^{\prime}$ as 0 separately and utilize and perform inverse transform given in equation (3) and (4) to recover the image.

b) If $\mathrm{X}^{\prime}$ is an even number, replace the LSB of ( $\left.\mathrm{X}^{\prime}, \mathrm{Y}^{\prime}\right)$ as 1 temporarily and carry into the equation 1 for calculation. If there is no overflow or underflow problem, it means that there is embedded message in this segment. Then, we replace the LSB of $\mathrm{X}^{\prime}$ and $\mathrm{Y}^{\prime}$ as 1 to recover the cover image.

c) If $\mathrm{X}^{\prime}$ is an even number and there is an overflow or underflow problem, it means that there is no embedded message in this segment. In order to restore the cover image, we utilize the previous recorded LSB of X to replace the LSB of $X^{\prime}$.

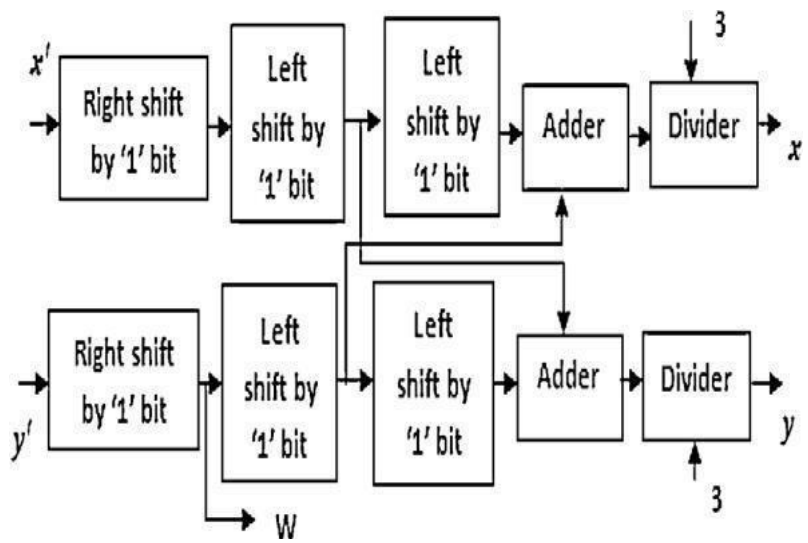

Fig 6. Data path for step-1 of watermark extraction 
ISO 3297:2007 Certified

Vol. 5, Issue 5, May 2017

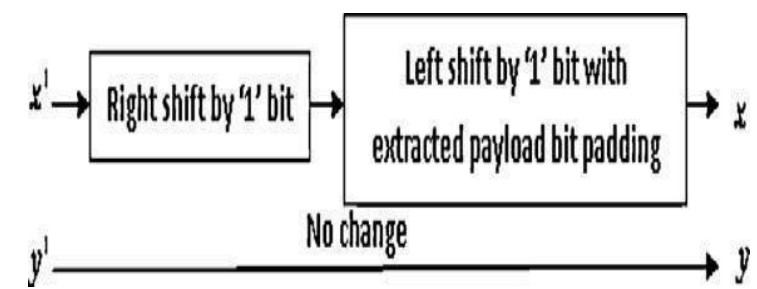

Fig 7. Data path for step-2 of watermark extraction

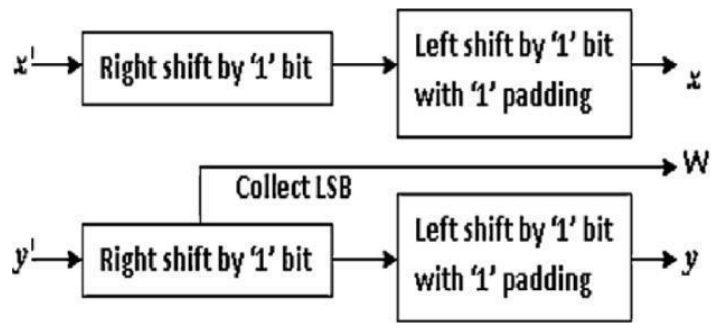

Fig 8. Data path for step-3 of watermark extraction

Fig 6. Illustrates that in step 1 for watermark extraction, the input is given as $\mathrm{x} 1$. Then it is left shifted by 1 bit for 2 times and it is added with the value $\mathrm{w}$, divided by 3 and the output will be $\mathrm{x}$. same process repeated for $\mathrm{y} 1$. Fig 7 illustrates that in step 2 for watermark extraction, the input is given as $\mathrm{x} 1$, and it is right shifted by 1 bit and the left shifted by 1 bit with 1 padding. The output will be same process is repeated for y and LSB also extracted. Fig 8 illustrates that in step 3 for watermark extraction, the input is given as $\mathrm{x} 1$ and it is right shifted by 1 bit and left shifted by 1 bit with extracted payload bit padding and the output will be x. Next y1 is given as input without change the output will be $y$.

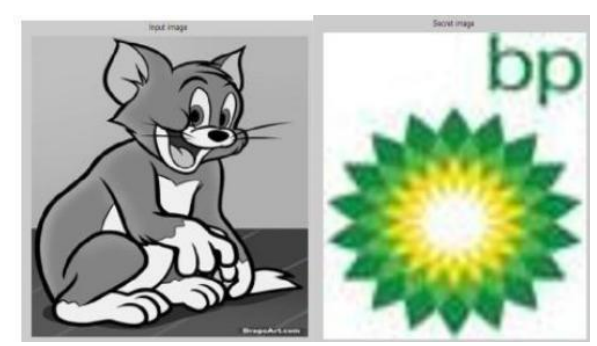

Fig 9. Input image Fig 10. Secret Image

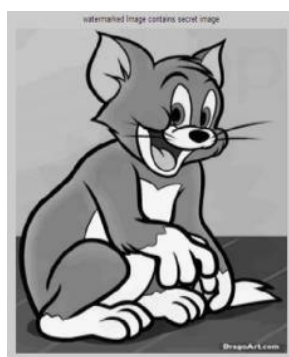

Fig 11. Watermarked image contains secret image

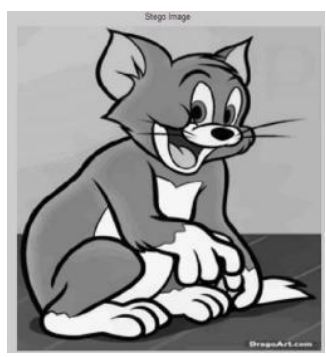

Fig 12. Watermarked image

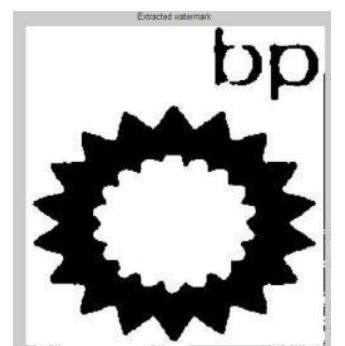

Fig 13. Extracted watermark 


\section{ISO 3297:2007 Certified}

Vol. 5, Issue 5, May 2017

\section{IV.HDL CODER}

A hardware description language enables a precise, formal description of an electronic circuit that allows for the automated analysis, simulation, and simulated testing of an electronic circuit. It also allows for the compilation of an HDL program into a lower level specification of physical electronic components, such as the set of masks used to create an integrated circuit. The HDL Workflow Advisor in HDL Coder automatically converts MATLAB code from floating-point to fixed-point and generates synthesizable VHDL and Verilog code This capability lets model the algorithm at a high level using abstract MATLAB constructs and System objects while providing options for generating HDL code that is optimized for hardware implementation. Fig 13 illustrates that the input image, Fig 10 illustrates that secret image, Fig 11 illustrates that watermark image contains secret image, Fig 12 illustrates that watermarked image, Fig 13 illustrates that extracted watermark, Fig 14 illustrates that semicustom layout. After generating HDL code, net list is created. By using net list, semicustom is implemented in mentor graphics

\section{CONCLUSION}

Reversible Watermarking (RW) algorithm is selected based on test image results. Performance gain in RCM-RW in terms of embedding bit rate (in bpp) and security of the hidden data are determined using simulation results of MATLAB. To improve the quality and performance of final image with respect to original image, the proposed algorithm reduces the calculation complexity of embedding and scaling factors. Future work may be extended for Xilinx ISE simulator by VLSI architecture of the watermarking algorithm of RCM-RW in FPGA environment for a gray scale image. It is also seen that the maximum combinational path delay during embedding process is calculated using Xilinx ISE simulator. The hardware implementation is quite simple, fast and requires less number of components for real time applications

\section{REFERENCES}

[1] Hirak Kumar Maitya,Santi P. Maityb "FPGA implementation of reversible watermarking in digital images using reversible contrast mapping", May 2014. value decomposition via artificial bee colony" in revised form 11 December 2014

[2] Santi P.Maity a,n, SebaMaity b, JayaSil a, ClaudeDelpha c"Perceptually adaptive MC-SS image watermarking using GA-NN hybridization in fading gain " December 2014.

[3]RashaThabit, BeeEeKhoo “ A new robust lossless data hiding scheme and its application to color medical images” December 2013.

[4]RashaThabit, BeeEeKhoo “A new robust lossless data hiding scheme and its application to color medical images" December 2013.

[5] Ehsan Nezha darya, Rabab K.Ward "Multiscale Derivative Transform and Its Application to Image Watermarking" July 2014.

[6].Linfei Chenn, Jingyu Liu, JisenWen, Xiong Gao, Haidan Mao Xiaoyan Shi, QinglingQu "A new optical image encryption method based on multi-beams interference and vector composition" 7 December 2014

[7.]XiaoyanLi a, HongjieHe a,n, ZhongkeYin a,b, FanChen a, JunCheng "KPLS-based image super-resolution using clustering and weighted boosting" 13 June 2015

[8].Shih-ChiehWei, Young-Chang Hou , Yen-Chun Lu Shih-ChiehWei, Young-Chang Hou,Yen-Chun Lu "A technique for sharing a digital image" 7 February 2015

[9].Min Qi , Bing-ZhaoLi , HuafeiSun "Image watermarking using polar harmonic transform with parameters in SL2;RP" 31 December 2015

[10].José Ramón Padilla-López , Alexandros Andre Chaaraoui , Francisco Flórez-Revuelta "Visual privacy protection methods: A survey" 31 January 2015

[11].Li Li , Zihui Dong, Jianfeng Lu , Junping Dai , Qianru Huang , Chin-Chen Chang, Ting Wu “AN H.264/AVC HDTV watermarking algorithm robust to camcorder Recording" 16 September 2014

[12].Musrrat Ali a, Chang Wook Ahn a, Millie Pant b, Patrick Siarry c "An image watermarking scheme in wavelet domain with optimized compensation of singular"20 November 2015 